\title{
Multi-Objective Optimization of Pilots' FFS Recurrent Training Problem
}

\author{
Mingang Gao \\ Institute of Policy and Management, Chinese Academy of Sciences, Beijing, China \\ Email:mggao@casipm.ac.cn
}

Received July 21, 2012; revised August 24, 2012; accepted September 3, 2012

\begin{abstract}
Two multi-objective programming models are built to describe Pilots' full flight simulator (FFS) recurrent training (PFRT) problem. There are two objectives for them. One is the best matching of captains and copilots in the same aircraft type. The other is that pilots could attend his training courses at proper month. Usually the two objectives are conflicting because there are copilots who will promote to captains or transfer to other aircraft type and new trainees will enter the company every year. The main theme in the research is to find the final non-inferior solutions of PFRT problem. Graph models are built to help to analyze the problem and we convert the original problem into a longest-route problem with weighted paths. An algorithm is designed with which we can obtain all the non-inferior solutions by a graphic method. A case study is present to demonstrate the effectiveness of the algorithm as well.
\end{abstract}

Keywords: PFRT Problem; Multi-Objective Programming; Bipartite Graph; Longest-Route Problem; Graphic Method

\section{Introduction}

With the rapid development of the air transportation, aviation safety has become an important issue in Chinese airline companies. FFS (full flight simulator) recurrent training is an important method to ensure airmen's flight safety. Pilots need to take FFS (full flight simulator) recurrent training every half a year in order to keep the pilot qualification in Chinese airways [1]. It allows two airmen to be trained in an FFS at the same time and the two men had better be a captain and a copilot for the best training effect. Moreover, an airman's fitting month for the training is from the fifth month to the seventh month after his last training. And the sixth month after his last training is his optimal training month because it is a resource waste for him to be trained in the fifth month and he will not get the best training effect when the training is implemented in the seventh month. To make the plan, we should take these two objectives into account. So PFRT problem is a multi-objective problem.

Pan et al. studied the problem and present a decomposition algorithm [1]. However, the algorithm is not a polynomial time algorithm. Liu [2] described the problem with multiple resource constraints and a Genetic Algorithm was designed to solve it. The algorithm is an approximation algorithm which cannot get the optimal results in general. PFRT problem with resource constraints is an NP-hard problem in theory [3].

Multi-objective programming involves recognition that the decision maker is responding to multiple objectives. Generally, the objectives are conflicting, so that not all objectives can simultaneously arrive at their optimal levels. An assumed utility function is used to choose appropriate solutions. Several fundamentally different utility function form $\mathrm{s}$ have been used in multi-objective models. These forms may be divided into three classes: lexicographic, multi-attribute utility and unknown utility. The lexicographic utility function specification assumes that the decision maker has a strictly ordered preemptive preference system among objectives with fixed target levels. Multi-attribute utility approaches allow tradeoffs between objectives in the attainment of maximum utility. The third utility approach involves an unknown utility function assumption. Here the entire Pareto efficient (nondominated) solution set is generated so that every solution is reported wherein one of the multiple objectives is as satisfied as it possibly can be without making any other objective worse off [4]. Many techniques have been developed to solve the multi-objective programming, such as tabu search [5,6], simulated annealing [7], and foremost evolutionary algorithms [8,9]. And other important publications on metaheuristics for multi-objective optimization include the work of Gandibleux et al. $[10,11]$.

PFRT problem is analyzed in this study. In part 2, we describe the problem with mathematical programming models. We generate graphs with which we transform the problem into a longest-route problem on weighted paths. 
A polynomial time algorithm is developed to solve PFRT problem. In part 3, a case study is given. In last part, we summarize the work.

\section{Models and Methodology}

\subsection{Mathematical Programming Models}

The two objectives of PFRT problem are conflicting and the problem can be described as programming (a) and (b).

$a_{i}$ : the number of the captains whose optimal training month is $i$.

$b_{i}$ : the number of the copilots whose optimal training month is $i$.

$a_{i, j}$ : the number of the captains whose optimal training month is $i$ and who will attend the training in month j.

$b_{i, j}$ : the number of the captains whose optimal training month is $i$ and who will attend the training in month $j$.

We describe the PFRT problem as the following multi-objective mathematical programming model:

Programming (a):

$$
\begin{aligned}
& \max f_{1} \\
& \max f_{2}
\end{aligned}
$$

where

$$
\begin{aligned}
& f_{1}=\sum_{i=1}^{6} \min \left(a_{i i}+a_{i-1, i}+a_{i+1, i}, b_{i i}+b_{i-1, i}+b_{i+1, i}\right) \\
& f_{2}=-\sum_{i=1}^{6}\left(a_{i, i+1}+a_{i, i-1}+b_{i, i+1}+b_{i, i-1}\right)
\end{aligned}
$$

Subject to

$$
\begin{aligned}
& a_{i}=a_{i, i-1}+a_{i, i}+a_{i, i+1}, \quad 2 \leq i \leq 5 ; \\
& a_{i}=a_{i, i}+a_{i, i+1}, \quad i=1 ; \\
& a_{i}=a_{i, i-1}+a_{i, i}, \quad i=6 ; \\
& b_{i}=b_{i, i-1}+b_{i, i}+b_{i, i+1}, \quad 2 \leq i \leq 5 ; \\
& b_{i}=b_{i, i}+b_{i, i+1}, \quad i=1 ; \\
& b_{i}=b_{i, i-1}+b_{i, i}, \quad i=6 ; \\
& a_{i, j}=b_{i, j}=0, i=0 \text { or } j=7 ;
\end{aligned}
$$

$a_{i}, b_{i}$ are positive integers and $a_{i, j}, b_{i, j}$ are all nonnegative integers.

The first objective of the programming is to maximize the number of captains who are matched with copilots. The other objective is to minimize the number of the pilots who will not be trained in their optimal training months. The Formulas (3)-(9) assure all pilots will be trained.

The general model for the problem can be described as:

\section{Programming (b):}

$$
\begin{aligned}
& \max f_{1} \\
& \max f_{2}
\end{aligned}
$$

Subject to

$$
\begin{aligned}
& a_{i}=a_{i, i-1}+a_{i, i}+a_{i, i+1}, \quad 2 \leq i \leq n-1 ; \\
& a_{i}=a_{i, i}+a_{i, i+1}, \quad i=1 ; \\
& a_{i}=a_{i, i-1}+a_{i, i}, \quad i=n ; \\
& b_{i}=b_{i, i-1}+b_{i, i}+b_{i, i+1}, \quad 2 \leq i \leq n ; \\
& b_{i}=b_{i, i}+b_{i, i+1}, \quad i=1 ; \\
& b_{i}=b_{i, i-1}+b_{i, i}, \quad i=n ; \\
& a_{i, j}=b_{i, j}=0, i=0 \text { or } j=n+1
\end{aligned}
$$

$a_{i}, b_{i}$ are all positive integer and $a_{i, j}, b_{i, j}$ are all nonnegative integer.

The key work of this research is to find the non-inferior solutions. We will find an initial non-inferior solution by two steps. Firstly, we consider the programming with a single objective as follows. Secondly we will convert get other non-inferior solution by a graphic method in part 2.2.

Programming (c):

$$
\max f_{1}
$$

Subject to the constraints (12)-(18).

Here let $P_{\max }$ denote the optimal value for this programming. Then we solve the following programming.

Programming (d):

$$
\max f_{2}
$$

Subject to the constraints (12)-(18) and

$$
f_{1}=P_{\max }
$$

Let $Q^{0}$ denote the optimal value for this programming and the optimal solutions is the initialization for the following graph models. We will give a graphic method to find all the other non-inferior solutions sequentially.

\subsection{Graph Models}

We draw a bipartite graph $G=(A, B)$ in Figure 1. The vertex $u_{i} \in A$ denotes the captains whose optimal training month is $i$. And the vertex $v_{i} \in B$ denotes the copilots whose optimal training month is $i$. Here $a_{i}=\left|u_{i}\right|$ denotes the module of $u_{i}$, i.e. the number of the captains in $u_{i}$ and $b_{i}=\left|v_{i}\right|$ denotes the module of $v_{i}$ and $q_{i}$ denotes the number of the pilots trained in their optimal training month $i$. There is an edge $u_{i} v_{j}$ weighted by $p_{i j}$ ended by $u_{i}$ and $v_{j}$ when there exist $p_{i j}$ pairs of captains and copilots we match between 


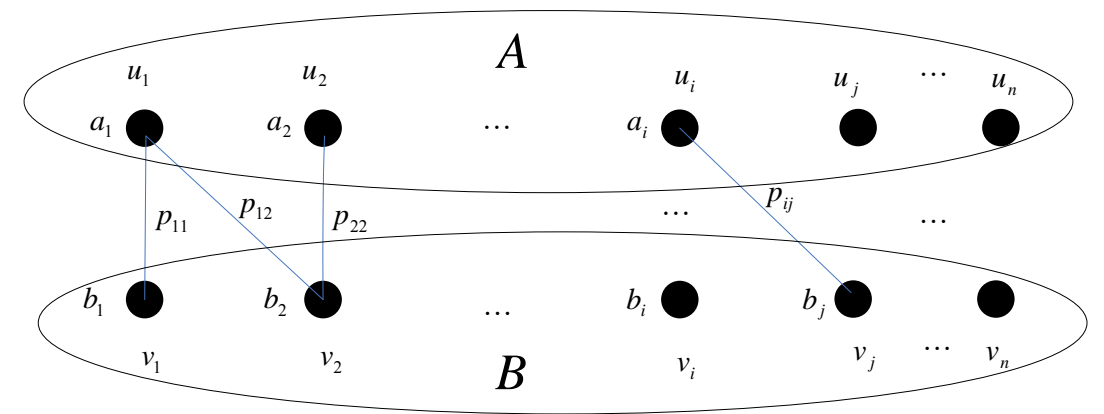

Figure 1. Bipartite graph $G$.

$u_{i}$ and $v_{j}$.

In graph $G$ there may be an edge $u_{i} v_{j}$ ended by $u_{i}$ and $v_{j}$ where $|i-j|=2$. In other words, there are a captain $\alpha_{i, k} \in u_{i}$ and a copilot $\beta_{j, s} \in v_{j}$ matched where $j=i+2$ (or $j=i-2$ ). Suppose $\alpha_{i+1, k^{\prime}} \in u_{i+1}$ (or $\left.\alpha_{i-1, k^{\prime}} \in u_{i-1}\right)$ and $\beta_{i+1, s^{\prime}} \in v_{i+1}\left(\beta_{i-1, s^{\prime}} \in v_{i-1}\right)$ are matched in graph $G$, we can change the training plan by matching $\alpha_{i, k}$ to $\beta_{i+1, s^{\prime}}$ and $\alpha_{i+1, k^{\prime}}$ to $\beta_{i+2, s}$ (or $\alpha_{i, k}$ to $\beta_{i-1, s^{\prime}}$ and $\alpha_{i-1, k^{\prime}}$ to $\left.\beta_{i-2, s}\right)$. Then we suppose there are no $u_{i} v_{j}$ ended by $u_{i}$ and $v_{j}$ where $|i-j|=2$ in the following part of the study.

Let $P=\sum_{i, j} p_{i, j}$ and $Q=\sum_{i} q_{i}$. What we will do in next parts of this study is to find the largest $Q$ when $P=P_{\max }$ is decreased by $k$ where $k=1,2, \cdots$.

We assume there are three possible nexus among $u_{i}$, $v_{i}, u_{i+1}$ and $v_{i+1}$ in graph $\mathrm{G}$ shown in Figure 2.

We consider two types to change the nexus among $u_{i}$, $v_{i}, u_{i+1}$ and $v_{i+1}$ in $G$ as follows. One is to break a pair on edge $u_{i} v_{i+1}$ in case 1 in Fiugre 3 or to break a pair on edge $u_{i} v_{i}$ and a pair on edge $u_{i+1} v_{i+1}$ and rebuilt a new pair between $u_{i+1}$ and $v_{i}$ in case 2 and case 3. It is denoted by $\operatorname{br}\left(u_{i}, v_{i+1}\right)$. The other is to break a pair on edge $u_{i+1} v_{i}$ in case 2 in Figure 3 or to break a pair on edge $u_{i} v_{i}$ and a pair on edge $u_{i+1} v_{i+1}$ and rebuilt a new pair between $u_{i}$ and $v_{i+1}$ in case 1 and 3 . It is denoted by $\operatorname{br}\left(u_{i+1}, v_{i}\right)$.

Then we can conclude that we can find the solution of the problem with $P=P_{\max }-1$ by $\operatorname{br}\left(u_{i}, v_{i+1}\right)$ or $\operatorname{br}\left(u_{i+1}, v_{i}\right)$ for all $1 \leq i \leq k$ where $1 \leq k \leq n-1$.

We generate another two weighted graphs $G^{1}$ and $G^{2}$ in Figure 3 from the recurrent training graph $G$ in Figure 1.

Here $v_{i}$ denotes month $i$ in Figure 3 and $l_{i}=1$ when there exist edge $u_{i} v_{i+1}$ in graph $G$, or else, $l_{i}=-1$. And $l_{i}^{\prime}=1$ when there exists edge $u_{i+1} v_{i}$, or else, $l_{i}^{\prime}=-1$. Let $L=\max _{\substack{1 \leq i \leq n-1 \\ i+1 \leq i+k \leq n-1}} \sum_{m=i}^{i+k} l_{m}=\sum_{m=i_{0}}^{i_{0}+k_{0}} l_{m}$ and $L^{\prime}=\max _{\substack{1 \leq i \leq n-1 \\ i+1 \leq i+k \leq n-1}} \sum_{m=i}^{i+k} l_{m}^{\prime}=\sum_{m=i_{0}^{\prime}}^{i_{0}^{\prime}+k_{0}^{\prime}} l_{m}^{\prime}$.
Then we have the follow theorem.

\section{Theorem 1:}

Let $G$ be the graph with $Q=Q^{0}$ and $P=P^{0}$, we got a non-inferior solution $p_{i, j}$ in graph $G^{\prime}$ with the largest $Q$ where $P=P^{0}-1$ when we change the nexus among vertices in graph $G$ by:

(a) $\operatorname{br}\left(u_{i}, v_{i+1}\right)$ for all $i_{0} \leq i \leq k_{0}$ when $L=\max \left\{L, L^{\prime}\right\}$ and

(b) $\operatorname{br}\left(u_{i+1}, v_{i}\right)$ for all $i_{0}^{\prime} \leq i \leq k_{0}^{\prime}$ when $L^{\prime}=\max \left\{L, L^{\prime}\right\}$.

\section{Proof:}

In case (a) we find that the nexus among the vertices $u_{i}, v_{i}, u_{i+1}$ and $v_{i+1}$ is case 1 in Figure 3 when $l_{i}=1$. When we change the nexus among $u_{i}, v_{i}, u_{i+1}$ and $v_{i+1}$ by $\operatorname{br}\left(u_{i}, v_{i+1}\right), p_{i, i+1}$ reduces one and the sum of $q_{i}$ and $q_{i+1}$ increases 1 , which equals to $l_{i}$, as an result. The nexus among $u_{i}, v_{i}, u_{i+1}$ and $v_{i+1}$ is case 2 or case 3 when $l_{i}=-1$, and when we change the nexus by $\operatorname{br}\left(u_{i}, v_{i+1}\right)$, we will get a new pair between $u_{i+1}$ and $v_{i}$ where $p_{i+1, i}=1$ and the sum of $q_{i}$ and $q_{i+1}$ is decreased by one and the sum of $p_{i, i}, p_{i+1, i+1}$ and $p_{i+1, i}$ is reduced by one as well, corresponding to $l_{i}$. In conclusion, when we change the nexus among $u_{i}$, $v_{i}, u_{i+1}$ and $v_{i+1}$ by $\operatorname{br}\left(u_{i}, v_{i+1}\right)$ we increase the sum of $q_{i}$ and $q_{i+1}$ by $l_{i}$ and increase $Q$ by $l_{i}$ in other words. Then to find the largest $Q$ where $P=P^{0}-1$ in graph $G$ is to find the longest path in graph $G^{1}$ when $L=\max \left\{L, L^{\prime}\right\}$. Case (b) can be proved similarly.

\subsection{Algorithm}

In this part an algorithm as follows is designed to solve PFRT problem.

Step 1: Initialize: $t=0$; for all $1 \leq i \leq n, a_{i}=$ the number of the captains whose optimal training month is $i$ and $b_{i}=$ the number of the copilots whose optimal training month is $i$.

Step 2: Find the optimal solutions of programming (c) and $(\mathrm{d}) . \quad G_{t}=\left(A_{t}, B_{t}\right)$ denotes the graph with $P^{t}=P_{\max }$ and $Q^{t}=$ the maximum quantity of pilots who will be trained in their optimal training month.

Step 3: Generate the weighted graphs $G_{t}^{1}$ and $G_{t}^{2}$ 


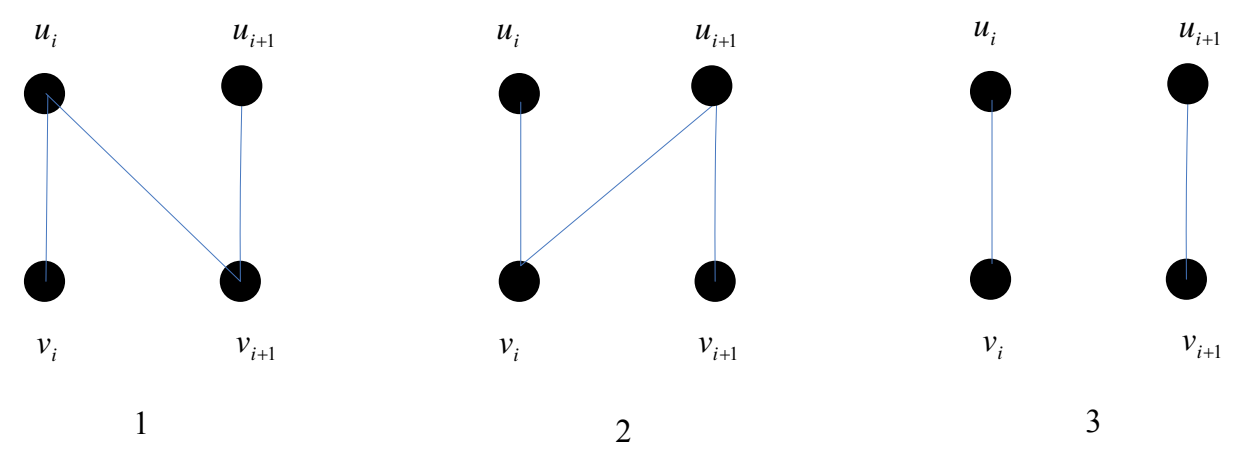

Figure 2. Nexus among $u_{i}, v_{i}, u_{i+1}$ and $v_{i+1}$ in graph $G$.
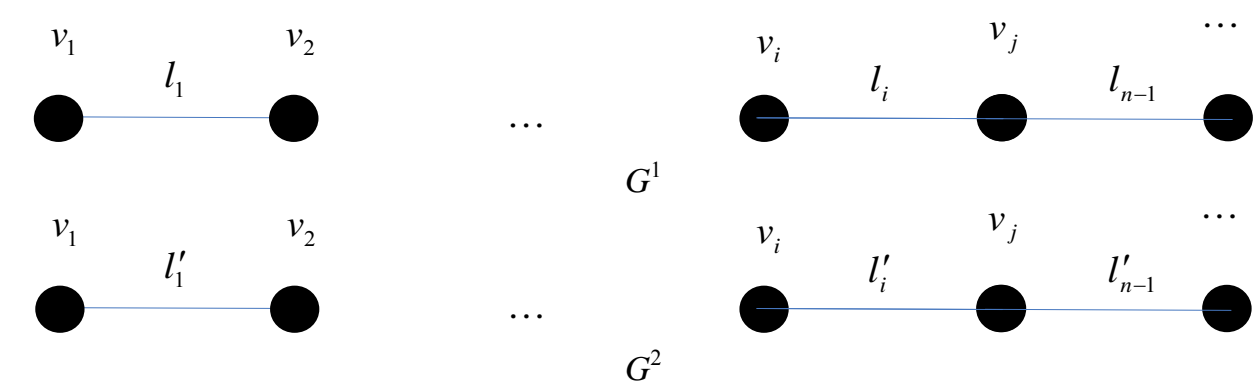

Figure 3. Generated graphs of $G$.

from the graph $G_{t}$. Find the longest path in the graphs

$G_{t}^{1}$ and $G_{t}^{2} \cdot L=\max _{\substack{1 \leq i \leq n-1 \\ i+1 \leq i+k \leq n-1}} \sum_{m=i}^{i+k} l_{m}=\sum_{m=i_{0}}^{i_{0}+k_{0}} l_{m}$ and

$L^{\prime}=\max _{\substack{1 \leq i \leq n-1 \\ i+1 \leq i+k \leq n-1}} \sum_{m=i}^{i+k} l_{m}^{\prime}=\sum_{m=i_{0}^{\prime}}^{i_{0}^{\prime}+k_{0}^{\prime}} l_{m}^{\prime}$. If $L \geq L^{\prime}$, go to step 4 .

Else go to step 5 .

Step 4: Change the nexus among the vertice $u_{i}, v_{i}$, $u_{i+1}$ and $v_{i+1}$ by $\operatorname{br}\left(u_{i}, v_{i+1}\right)$ for $i_{0} \leq i \leq i_{0}+k_{0}-1$. Go to step 6 .

Step 5: Change the nexus among the vertices $u_{i}, v_{i}$, $u_{i+1}$ and $v_{i+1}$ by $\operatorname{br}\left(u_{i+1}, v_{i}\right)$ for $i_{0}^{\prime} \leq i \leq i_{0}^{\prime}+k_{0}^{\prime}-1$. Go to step 6.

Step 6: If the non-inferior solution we got in the previous step is not the final solution, let $t=t+1$, $P_{t}=P_{t-1}-1$, go to step 3, else go to step 7 .

Step 7: Output the final solution, stop.

\section{A Case Study}

There are 314 captains and 313 copilots in an airline company. Their FFS training demand is shown in Table 1 and $P^{0}=P_{\max }=313$ is achieved in Table 2 and Figure 4 where $Q^{0}=\sum_{i=1}^{6} q_{i}=609$. We paired up 313 pairs of captains and copilots. 609 pilots will attend FFS training in their optimal training months. There are 18 pilots who have to attend the training in the previous month or the succeeding month of their optimal training months.
Table 1. FFS training demand for all pilots

\begin{tabular}{ccc}
\hline (Month) & $\begin{array}{c}a_{i} \text { (The number of captains } \\
\text { need to be trained in } \\
\text { month } i \text { ) }\end{array}$ & $\begin{array}{c}b_{i} \text { (The number of copilots } \\
\text { need to be trained in } \\
\text { month } i \text { ) }\end{array}$ \\
\hline 1 & 51 & 55 \\
2 & 50 & 49 \\
3 & 48 & 51 \\
4 & 60 & 56 \\
5 & 55 & 49 \\
6 & 50 & 53 \\
\hline
\end{tabular}

Table 2. $P^{0}=P_{\max }=313$ and $Q^{0}=\sum_{i=1}^{6} q_{i}=609$.

\begin{tabular}{|c|c|c|c|c|c|c|c|c|}
\hline & $i$ & 1 & 2 & 3 & 4 & 5 & 6 & $b_{j}$ \\
\hline$j$ & \multicolumn{8}{|c|}{$P_{i, j}$} \\
\hline 1 & & 51 & 4 & & & & & 55 \\
\hline 2 & & & 46 & 3 & & & & 49 \\
\hline 3 & & & & 45 & 6 & & & 51 \\
\hline 4 & & & & & 54 & 2 & & 56 \\
\hline 5 & & & & & & 49 & & 49 \\
\hline 6 & & & & & & 3 & 50 & 53 \\
\hline Not matched & & & & & & 1 & & \\
\hline$a_{i}$ & & 51 & 50 & 48 & 60 & 55 & 50 & \\
\hline
\end{tabular}




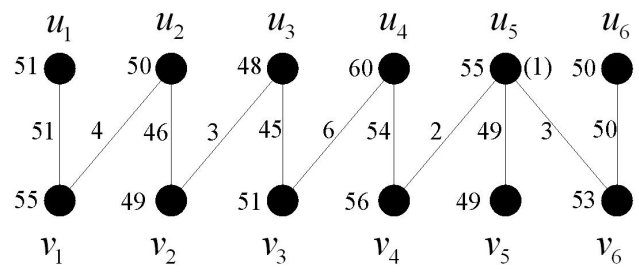

Figure 4. The initial non-inferior solution with $P^{0}=313$ in graph $G_{0}$.

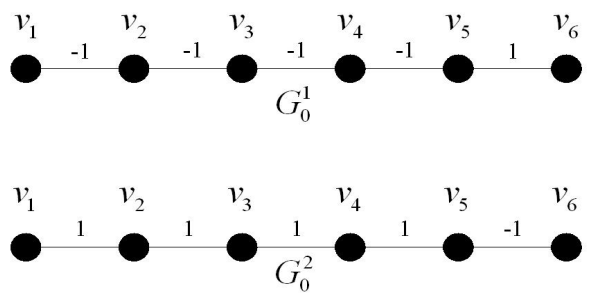

Figure 5. Generated graphs of $\boldsymbol{G}_{0}$.

And there is one captain in month 5 who cannot be trained with copilots because the sum of captains is more than that of copilots. lows.

We draw a couple of new graphs $G_{0}^{1}$ and $G_{0}^{2}$ as fol-

We can find that the longest route in graph $G_{0}^{1}$ is $v_{5} v_{6}$ where $L=1$ and the longest route in graph $G_{0}^{2}$ is $v_{1} v_{2} v_{3} v_{4} v_{5}$ where $L=4$. We change the nexus among $u_{1}$, $v_{1}, u_{2}$ and $v_{2}$ by $\operatorname{br}\left(u_{2}, v_{1}\right)$. Then $p_{2,1}$ will reduce one and $P=\sum_{i, j} p_{i, j}$ will reduce one as well. In the same time $\sum_{i} q_{i}$ will increase one. When we change the nexus among $u_{i}, v_{i}, u_{i+1}$ and $v_{i+1}$ by $\operatorname{br}\left(u_{i+1}, v_{i}\right)$ as well where $1 \leq i \leq 4$ and rematch the pairs of unmatched captains and copilots who have the same optimal training month, we will get a new graph $G_{1}$ with the largest $Q=Q^{1}$ where $P^{1}=\sum_{i, j} p_{i, j}=P^{0}-1=312$ and $Q^{1}=613$. Then we get a new non-inferior solution described in Table 3.

Now we paired up 312 pairs of captains and copilots. 613 pilots will attend FFS training in their optimal training months. There are 14 pilots who have to attend the training in the previous month or the succeeding month of their optimal training months. And there are 3 pilots in month 5 and month 1 who cannot be matched with other pilots.

Repeating above steps, we will get the following solutions shown in Tables 4-6.

Now we get all non-inferior solutions.

\section{Conclusions}

The air transportation developed rapidly in China and it becomes crucially important for airline companies to
Table 3. $P^{1}=312$ and $Q^{1}=613$.

\begin{tabular}{ccccccccc}
\hline & $i$ & 1 & 2 & 3 & 4 & 5 & 6 & $\begin{array}{c}\text { Not } \\
\text { matched }\end{array}$ \\
\hline$j$ & & & & $P_{i, j}$ & & & \\
1 & 51 & 3 & & & & & 1 & 55 \\
2 & & 47 & 2 & & & & & 49 \\
3 & & & 46 & 5 & & & & 51 \\
4 & & & & 55 & 1 & & 56 \\
5 & & & & & 49 & & 49 \\
6 & & & & & 3 & 50 & \\
Not matched & & & & & 2 & & & \\
$a_{i}$ & 51 & 50 & 48 & 60 & 55 & 50 & & \\
\hline
\end{tabular}

Table 4. $P^{1}=311$ and $Q^{1}=617$.

\begin{tabular}{ccccccccc}
\hline & $i$ & 1 & 2 & 3 & 4 & 5 & 6 & $\begin{array}{c}\text { Not } \\
\text { matched }\end{array}$ \\
\hline$j$ & & & & $P_{j}$ & \\
1 & 51 & 2 & & & & & 2 & 55 \\
2 & & 48 & 1 & & & & & 49 \\
3 & & & 47 & 4 & & & & 51 \\
4 & & & & 56 & & & 56 \\
5 & & & & & 49 & & \\
6 & & & & & 3 & 50 & \\
Not matched & & & & & 3 & & \\
$a_{i}$ & 51 & 50 & 48 & 60 & 55 & 50 & & \\
\hline
\end{tabular}

Table 5. $P^{1}=310$ and $Q^{1}=620$.

\begin{tabular}{|c|c|c|c|c|c|c|c|c|}
\hline & $\begin{array}{ll}i & 1\end{array}$ & 2 & 3 & 4 & 5 & 6 & $\begin{array}{c}\text { Not } \\
\text { matched }\end{array}$ & $b_{j}$ \\
\hline$j$ & \multicolumn{8}{|c|}{$P_{i, j}$} \\
\hline 1 & 51 & 1 & & & & & 3 & 55 \\
\hline 2 & & 49 & & & & & & 49 \\
\hline 3 & & & 48 & 3 & & & & 51 \\
\hline 4 & & & & 56 & & & & 56 \\
\hline 5 & & & & & 49 & & & 49 \\
\hline 6 & & & & & 3 & 50 & & 53 \\
\hline Not matched & & & & 1 & 3 & & & \\
\hline$a_{i}$ & 51 & 50 & 48 & 60 & 55 & 50 & & \\
\hline
\end{tabular}

Table 6. $P^{1}=310$ and $Q^{1}=627$.

\begin{tabular}{ccccccccc}
\hline & $i$ & 1 & 2 & 3 & 4 & 5 & 6 & $\begin{array}{c}\text { Not } \\
\text { matched }\end{array}$ \\
\hline$j$ & & & & $P_{j}$ & \\
1 & 51 & & & & & & 4 & 55 \\
2 & & 49 & & & & & & 49 \\
3 & & & 48 & & & & 3 & 51 \\
4 & & & & 56 & & & & 56 \\
5 & & & & & 49 & & & 49 \\
6 & & & & & & 50 & 3 & 53 \\
Not matched & & 1 & & 4 & 6 & & & \\
$a_{i}$ & 51 & 50 & 48 & 60 & 55 & 50 & & \\
\hline
\end{tabular}


training pilots so as to ensure the aviation safety.

PFRT problem is a multi-objective pilots training problem. Mathematic models and graph models about it are built. According to the characteristics of the problem, we convert it into a longest-route problem with weighted paths and design an algorithm to solve it.

This method can effectively generate pilots' FFS training plans with two kinds of personnel.

Due to the method in this study cannot solve the similar problem involving more than two kinds of personnel yet, further research should be done on it.

\section{Acknowledgements}

I thank members of the Research Center of Overall Planning Safety and Security Management, Institute of Policy and Management, Chinese Academy of Sciences, for their help in this study. We are also very grateful to the reviewers for their invaluable suggestions to improve the quality of the manuscript.

\section{REFERENCES}

[1] B. C. Pan, Z. Y. Song, et al., "The Research on Optimization Model for Recurrent Training Plan of Aviator Full Flight Simulators," Chinese Journal of Management Science, Vol. 16, No. 2, 2008, pp. 69-75.

[2] W. B. Liu, S. Z. Zhang and B. L. Shi, "Solution of Pilot Simulator Timetable Problem Based on Genetic Algorithm," Computer Engineering, Vol. 37, No. 15, 2011, pp. 140-142.

[3] I. H. Toroslu and Y. Arslanoglu, "Genetic Algorithm for the Personnel Assignment Problem with Multiple Objectives," Information Sciences, Vol. 177, No. 3, 2007, pp.
787-803. doi:10.1016/j.ins.2006.07.032

[4] A. M. Geoffrion, "Proper Efficiency and the Theory of Vector Maximization," Journal of Mathematical Analysis and Applications, Vol. 22, No. 3, 1968, pp. 618-630. doi:10.1016/0022-247X(68)90201-1

[5] J. F. Cordeau and M. Maischberger, "A Parallel Iterated Tabu Search Heuristic for Vehicle Routing Problems," Computers \& Operations Research, Vol. 39, No. 9, 2012, pp. 2033-2050. doi:10.1016/j.cor.2011.09.021

[6] Q. H. Wu, J. K. Hao, et al., "Multi-Neighborhood Tabu Search for the Maximum Weight Clique Problem," Annals of Operations Research, Vol. 196, No 1, 2012, pp. 611-634. doi:10.1007/s10479-012-1124-3

[7] R. Kia, A. Baboli, et al., "Solving a Group Layout Design Model of a Dynamic Cellular Manufacturing System with Alternative Process Routings, Lot Splitting and Flexible Reconfiguration by Simulated Annealing," Computers \& Operations Research, Vol. 39, No. 11, 2012, pp. 26422658. doi:10.1016/j.cor.2012.01.012

[8] K. Deb, "Multi-Objective Optimization Using Evolutionary Algorithms," New York, 2001.

[9] M. Cruz-Ramirez, C. Hervas-Martinez, et al., "MultiObjective Evolutionary Algorithm for Donor-Recipient Decision System in Liver Transplants," European Journal of Operational Research, Vol. 222, No. 2, 2012, pp. 317-327. doi:10.1016/j.ejor.2012.05.013

[10] A. Thapar, D. Pandey, et al., "Satisficing Solutions of Multi-Objective Fuzzy Optimization Problems Using Genetic Algorithm," Applied Soft Computing, Vol. 12, No. 8, 2012, pp. 2178-2187. doi:10.1016/j.asoc.2012.03.002

[11] E. G. Talbi, M. Basseur, et al., "Multi-Objective Optimization Using Metaheuristics: Non-Standard Algorithms," International Transactions in Operational Research, Vol. 19, No. 1-2, 2012, pp. 283-305. 